\title{
AC 2009-1772: AN INVESTIGATION OF WING MORPHING PHENOMENA IN THE EDUCATIONAL WIND TUNNEL
}

\section{B. Terry Beck, Kansas State University}

Terry Beck is a Professor of Mechanical and Nuclear Engineering at Kansas State University (KSU) and teaches courses in the fluid and thermal sciences. He conducts research in the development and application of optical measurement techniques, including laser velocimetry and laser-based diagnostic testing for industrial applications. Dr. Beck received his B.S. (1971), M.S. (1974), and Ph.D. (1978) degrees in mechanical engineering from Oakland University.

\section{Bill Whitson, Kansas State University}

Bill Whitson is a December 2008 recent graduate from the Mechanical and Nuclear Engineering Department at Kansas State University (KSU). He worked on the wing morphing test setup as part of a senior honors research project.

\section{Greg Payne, Kansas State University}

Greg Payne is a senior in the Mechanical and Nuclear Engineering Department at Kansas State University (KSU). In addition to his work as laboratory assistant on our MNE wind tunnel facility, where he has contributed significantly to wind tunnel lab development projects such as the current smoke rake and wing morphing project, he was also the team leader for the KSU SAE Aero Design Competition in 2008.

\section{Trevor Heitman, Kansas State University}

Trevor Heitman is a junior in the Mechanical and Nuclear Engineering Department at Kansas State University (KSU). He worked on the wing morphing project as part of his wind tunnel laboratory assistant activities, and has also contributed significantly to previous wind tunnel lab development projects including the current smoke rake system design. 


\title{
An Investigation of Wing Morphing Phenomena in the Educational Wind Tunnel
}

\begin{abstract}
Wing morphing is an important technique currently under investigation to reduce drag as well as noise associated with the design of aircraft. The general term "morphing" includes use of a variety of control mechanisms to reduce drag by altering the air flow around the surface of an aerodynamic shape. This would in general include the direct alteration of the shape of an aerodynamic surface, as well as the use of localized jets or other surface structures to likewise dynamically influence the flow. Bird flight, is one example of morphing which occurs in nature, and the highly flexible adaptive control exhibited by birds in flight is really quite remarkable. It would be desirable to mimic such behavior in order to improve practical aerodynamic performance. Of primary engineering interest is the control of so-called boundary layer separation, which is characterized by streamlines breaking away from the aerodynamic surface, ultimately leading to the onset of "stall" or loss of lift in the case of a wing surface, as well as severe increase in drag. Such phenomena can be studied both qualitatively and quantitatively using a wind tunnel or water tunnel test facility. Wind and water tunnel testing has long been an important component common to many introductory fluid mechanics and aerodynamics courses, but usually involves steady-state measurements of lift, drag, pitching moment, and pressure distribution on small-scale models. With morphing, there is an added "dynamic" dimension to the flow structure, which is characteristic of unsteady aerodynamics. This involves time dependent boundary layer separation and vortex shedding phenomena. A long-range goal would be to actively control this unsteady boundary layer separation to reduce the overall drag force, but not at the expense of too much complexity or the need for significant expenditure of energy. Understanding these physical characteristics is very important to future aerodynamic design, for the purpose of maximizing fuel economy. This area also represents an important topic associated with the teaching of basic principles of modern aerodynamic design as applied to existing aircraft control structures, such as flaps or elevator control surfaces.

The work presented here is the outgrowth of a one semester senior undergraduate mechanical engineering student honors research project, which makes use of our Aerolab educational wind tunnel test facility. The focus of this paper is to demonstrate basic characteristics of one type of wing morphing on a dynamic wing model, inexpensively fabricated using a rapid prototyping facility. Flow visualization of vortex shedding phenomena is illustrated for the dynamic wing morphing design. The pivoted wing structure test model is driven in periodic rotational motion by a mechanism designed by the associated honors student. This mechanical setup is used in conjunction with a recently developed special-purpose aerodynamic smoke rake system, and a relatively inexpensive low-power laser-based lighting system, for visualization of the associated air flow and vortex shedding phenomena.
\end{abstract}




\section{Introduction}

Flow visualization has long been used in conjunction with either smoke injection, for wind tunnel applications, or dye injection for water tunnel applications. The educational wind tunnel represents an important tool for introducing engineering students to basic experimental measurements of lift, drag, pitching moment, and pressure distribution (or pressure coefficient), usually first encountered in the setting of an introductory course in aerodynamics or a junior level course in fluid mechanics. In addition to providing quantitative aerodynamic measurements, the wind tunnel and its companion the water tunnel offer the ability to visualize the important physical characteristics of fluid flows. In the case of wind tunnel flow visualization, smoke injection is the usual means to visualize the flows; whereas with water tunnel flow visualization a dye injection method is typically employed. The ability to visualize flows, and the associated boundary layer separation and vortex shedding phenomenon, forms an extremely important thrust of such introductory courses, and is an invaluable educational tool as well as a tool for practical engineering design.

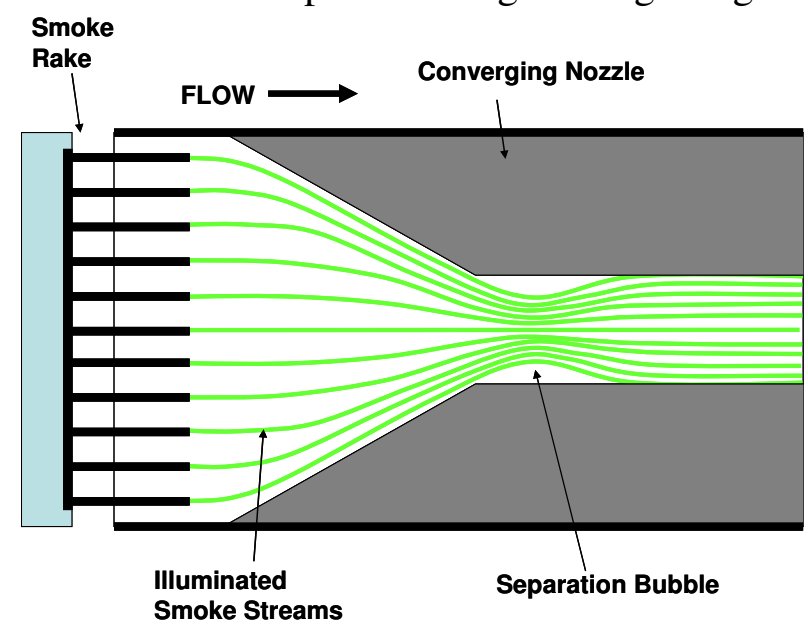

(a) Schematic of Sharp-Edged Nozzle Flow

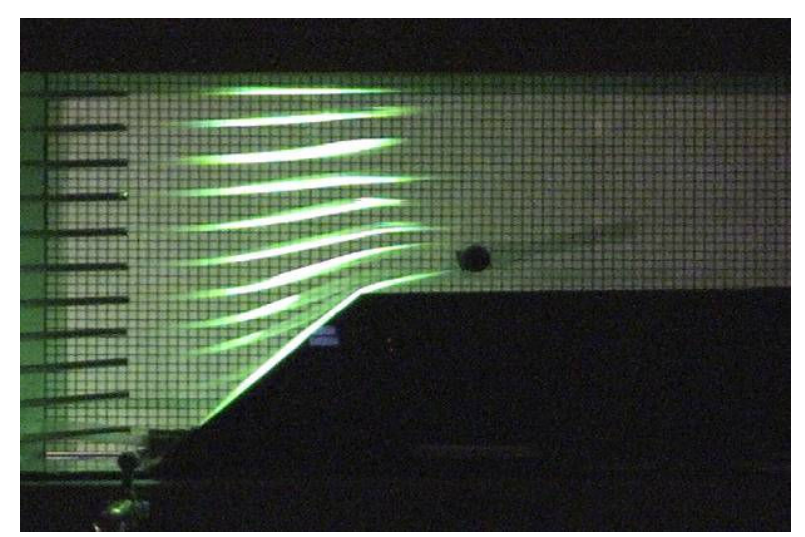

(b) Actual Half-Section Nozzle Flow

\section{Figure 1: Wind Tunnel Flow Visualization-Internal Flow}

The work presented here is also an outgrowth of a simple means to modify existing educational wind tunnel facilities to successfully demonstrate certain duct flow (stream tube) principles related to wind tunnel design ${ }^{1}$. It was shown that the entire wind tunnel could be used to further illustrate these important design principles. Recent developments in smoke flow visualization on our educational wind tunnel further extended this concept, by enabling full-field visualization of internal flow, such as is shown in Figure 1 for a sharp-edged converging nozzle ${ }^{2}$. An inexpensive method of constructing simple and functional internal duct models was presented, which could be incorporated into virtually any educational wind tunnel facility. In addition, a simple design for a general purpose smoke rake was introduced and tested with good results. The connection between diffuser frictional loss behavior and boundary layer separation phenomena associated with the re-circulating flows in the observed separation bubble is also brought out in these experiments. Eventually, a modular smoke rake is envisioned which can be adapted to several different test model geometries. A second generation design of one such portable rake system will be presented in this paper. This type of equipment, and the applications presented here, makes possible the visualization of a wide variety of flow 
phenomena in both internal and external flows, without the need of a special-purpose visualization flow tunnel.
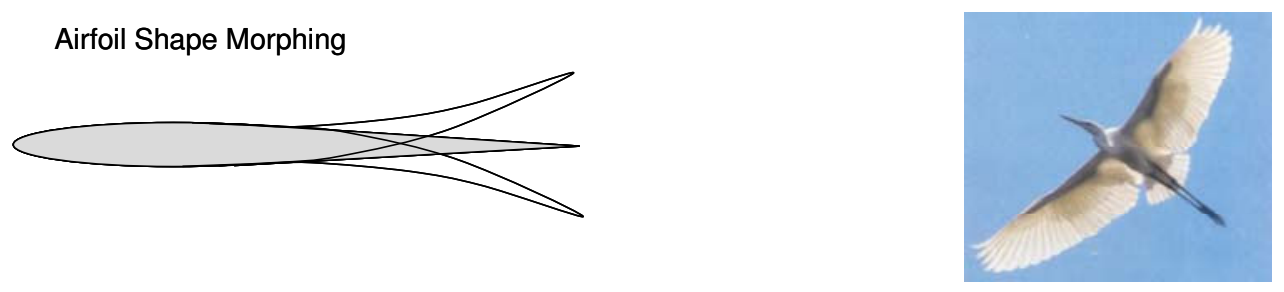

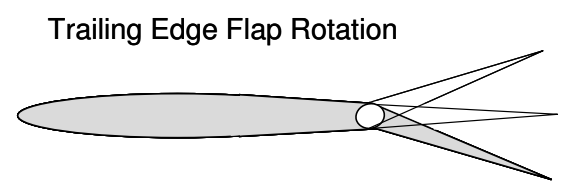

(a) Morphing of Airfoil Shape

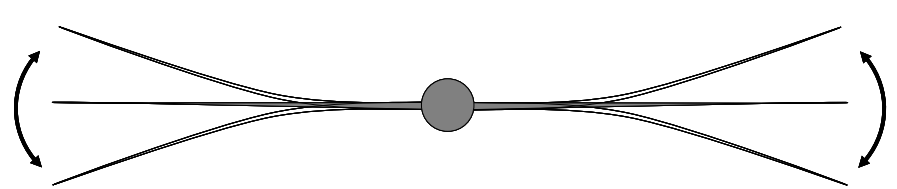

(b) Morphing of Entire Wing

\section{Figure 2: Various Wing Morphing Phenomena}

The objective of this paper is to further advance the adaptation of wind tunnel facilities to allow visualization of wing morphing phenomena. In addition, this work involves mentoring an undergraduate mechanical engineering student as part of a senior honors program design project. Wing morphing includes a broad spectrum of phenomena, from flexible alteration of airfoil shape to flapping phenomena similar to bird flight ${ }^{3}$, as suggested in Figure 2. A major motivation for such devices is to actively control unsteady boundary layer separation to reduce the overall drag force. Boundary layer control can be broadly classified into passive and active control $^{4}$. Common applications include the control of boundary layer separation in external flows to maintain the effectiveness of flaps and other control surfaces, and internal flow boundary layer control associated with the operation of jet engine inlet and diffuser flows. Passive control includes the use of common devices such as vortex generator tabs ${ }^{5}$ to delay the onset of separation associated with ailerons. Active control includes the many leading and trailing edge devices used in conjunction with flow control over wing surfaces ${ }^{6}$. Actuators of various types play an important role in active boundary layer control. A variety of different actuator devices have previously been investigated as flow control devices for both internal and external boundary layer applications. The operating principles for such devices are typically based on either mechanical deflection, mass injection, or through the use of synthetic jets ${ }^{7}$. Mechanical actuator devices include conventional flap controls, as well as more recent MEMS flap devices. A new type of combustion-driven pulsed-jet actuator has been proposed ${ }^{8,9}$ that would be capable of providing both high impulse and high frequency. Use of a flexible wall to produce a traveling wave for flow control has also been investigated ${ }^{10}$.

For the current work, a simplified periodically rotating trailing edge flap will be used to simulate the airfoil shape morphing shown in Figure 2(a). The modifications to the existing wind tunnel facility take the form of a simple externally mounted rotation mechanism. This inexpensive setup could be adapted to most modest wind tunnel facilities, using available parts from a local hardware store. To visualize the streamline flow, an improved simple smoke rake design similar to that used in previous flow visualization testing ${ }^{2}$ has also been designed to produce a set of uniformly spaced smoke streams. This smoke rake is easily introduced into the inlet of the test section region, upstream of the rotating wing model. Features associated with the smoke rake 
design and it's associated smoke injection system are presented along with the characteristics of the rotation mechanism. The previously developed laser illumination and streamline image capture system is described, along with a relatively simple camcorder image acquisition setup for filming the streamline flows. The streamline flow visualization results are illustrated for a symmetrical airfoil shape with a $40 \%$ trailing edge flap. This is but one of the basic flap geometries that are currently under investigation for this project. The undergraduate honors student was involved with all phases of the work, with major focus on the design, construction and demonstration of the rotation mechanism. Rapid prototyping was used for construction of the basic wing and flap assembly.

\section{Wind Tunnel Facility}

Figure 3 shows the existing Educational Wind Tunnel associated with the current development. A photograph of the overall facility is shown in Figure 3(a), and a longitudinal view of the wind tunnel showing the test section and instrumentation for data acquisition is shown in Figure 3(b).

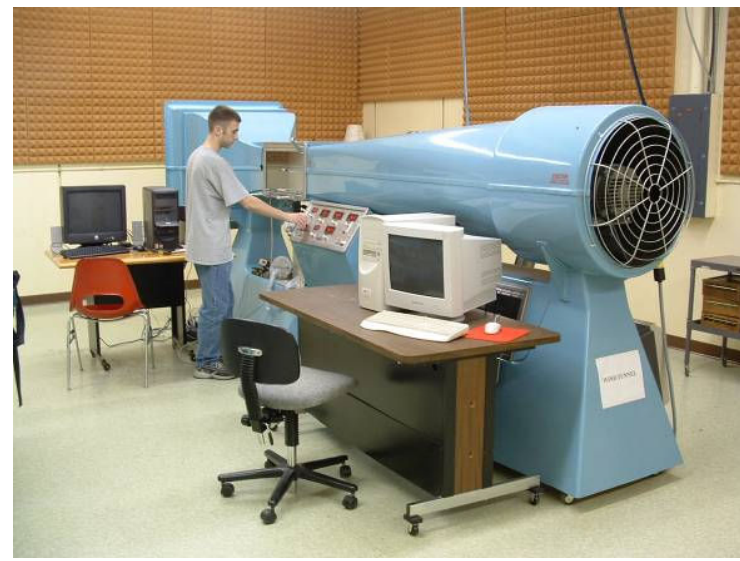

(a) Wind Tunnel Facility

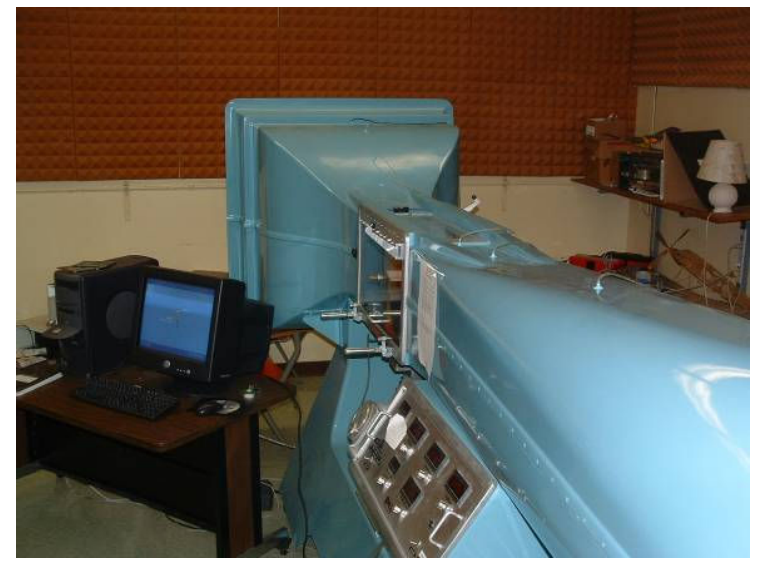

(b) Test Section and Instrumentation

\section{Figure 3: Educational Wind Tunnel Facility}

While relatively inexpensive in comparison to some wind tunnels, this facility has been demonstrated to be capable investigating a wide variety of phenomena of interest to fluid mechanics and aerodynamic courses. ${ }^{1,2,11,12}$ The wind tunnel has a test section measuring approximately 12 in x 12 in x 24 in $(305 \mathrm{~mm} \times 305 \mathrm{~mm} \times 610 \mathrm{~mm})$, and has a maximum air speed of approximately $140 \mathrm{mph}(63 \mathrm{~m} / \mathrm{s})$. It is instrumented with an electronic strain-gage based balance for measurements of normal force, axial force, pitching moment, and pressure distribution as a function of air speed and angle of attack. Both manual as well as electronic pressure sensing is available on this facility. An electronic pressure scanning unit containing 32 individual electronic pressure sensors is also used in conjunction with this facility. This latter unit can be used to provide real-time visualization of the pressure distribution in the wind tunnel, in much the same manner as it has been used to visualize the pressure distribution associated with airfoils and wings. ${ }^{11,13}$ Manual measurements are accessible from a front panel digital display shown in Figure 3(b), and electronic data acquisition is also available for remote access and real-time measurements. A recently developed flow visualization setup will be used for the purpose of the current morphing investigation, along with an improved smoke rake design. Key to the flow visualization system is the smoke rake and the associated smoke distribution system 
which are also described below.

\section{Smoke Rake System}

Smoke rakes are for some reason not readily available commercially for the existing educational wind tunnel facility. Hence, a general-purpose smoke rake flow visualization system was designed for our facility, and should also be applicable for use in similar wind tunnels. Figure 4 is a CAD drawing of basic the smoke rake developed and used in the current investigation. Figure 4(a) shows a side view and isometric view of the current rake design. The rake is modular and can support a variable number of tubes by adjusting the housing. In the current version used for testing presented below, the rake has a total of eighteen $1 / 4$-inch $(6.4 \mathrm{~mm})$ O.D stainless steel tubes. Smaller or larger tubes can be used, depending on the size of the test model. This size provides reasonable resolution of the flow fields associated with the morphing wing wake flows currently under investigation.

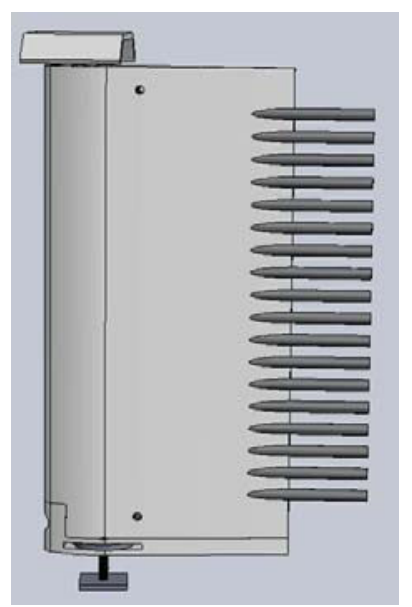

(a) Side and Isometric View

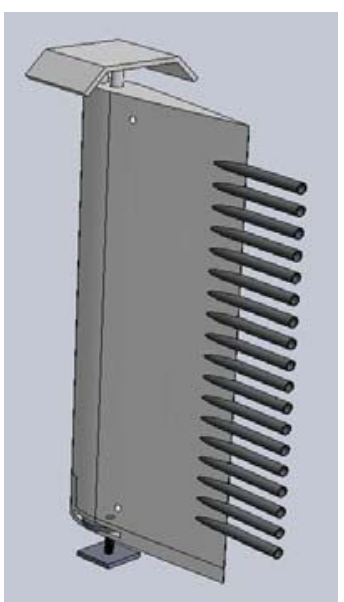

\section{Figure 4: New Smoke Rake Assembly}

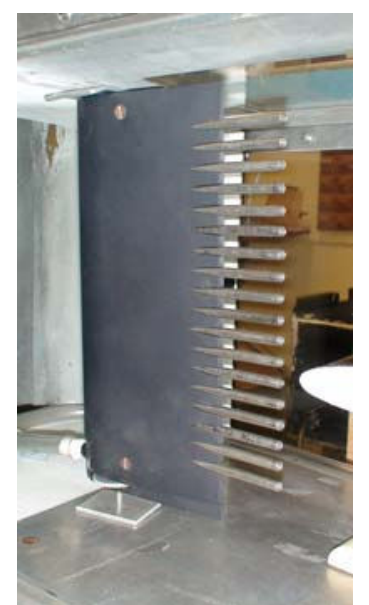

(b) Photograph in Wind Tunnel

There are a number of important issues to be considered in the design of a smoke rake flow visualization system, and a complete discussion and engineering analysis of all of these in detail could easily form an additional paper. Some of the main issues include the following:

- Number of streams and diameter of tubes.

- Length of tubes and radius of bend to align with flow.

- Design of manifold assembly, housing, and mounting of tubes.

- Balancing of injected smoke flow with outer flow stream.

- Level of turbulence in wind tunnel.

- Flow development inside the smoke rake tubes.

- External boundary layer development on smoke rake tubes.

- Tube wall thickness and geometry of exit port.

- Mounting of the rake in the wind tunnel test section.

To provide good flow visualization and streamline definition, laminar flow in the rake tubes is highly desirable. Otherwise, the smoke streams will break up very quickly after exiting the 
tubes, which will "wash out" the desired streamlines. Well defined streams on the order of 12 inches $(30 \mathrm{~cm})$ or more are possible with careful attention to this laminar flow requirement, and with proper balancing of the flow streams. The diameter and length (in external flow direction) of the flow injection tubes also limits the tube spacing. Too close and the external boundary layers from adjacent tubes will interfere, and also tend to block off the flow around the entire rake assembly. The current rake has a symmetrical airfoil design, which offers an improvement over the design used in the earlier testing shown in Figure 1 by significantly reducing flow disturbance caused by the housing. The housing was constructed from ABS plastic using our rapid prototyping facility.

\section{Smoke Generation System}

In addition to the rake itself, a smoke generation and smoke distribution system is required to supply smoke at a constant rate to the rake assembly. A schematic diagram of the current smoke distribution system is shown in Figure 5. Flow must also be provided through the test section. The flow velocities required to provide well-defined streamlines are considerable below the normal wind tunnel airspeeds. Hence, an external box-fan was positioned at the discharge end of the wind tunnel to suck air and provide the flow.

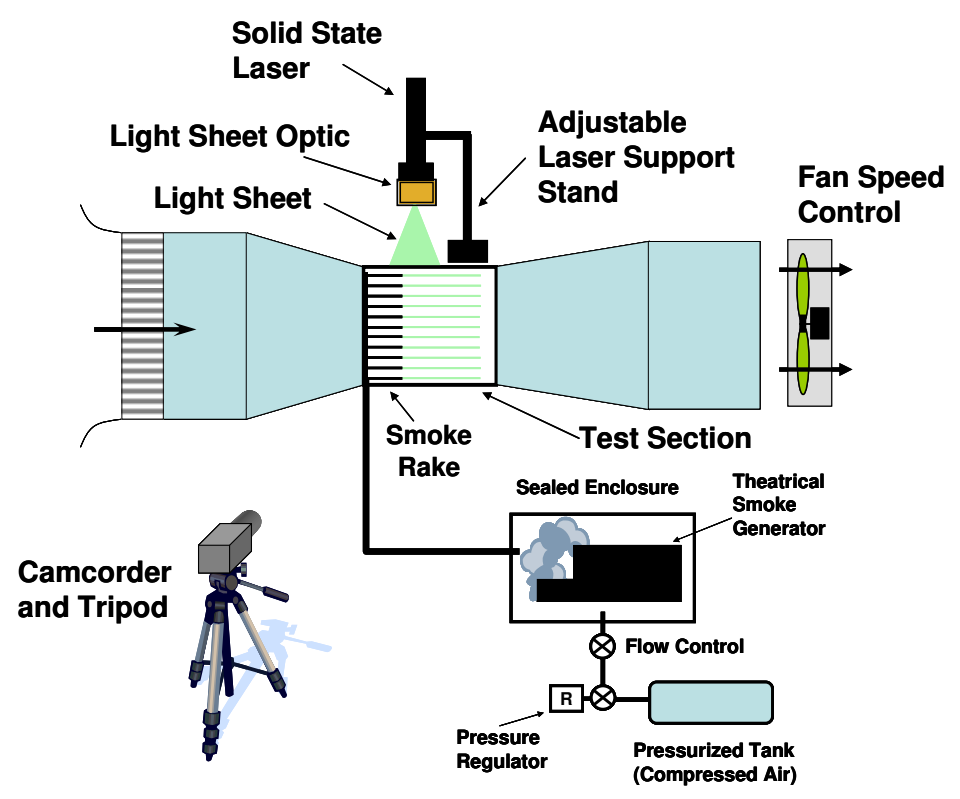

Figure 5: Smoke Distribution System

A Variac (variable transformer) was used to regulate the speed of this fan, and the relative spacing between the fan and the exit plane of the wind tunnel also provided some speed adjustment. In practice, a spacing of about 6-12 inches $(15-30 \mathrm{~cm})$ was sufficient for testing of the current wing morphing configurations. Another issue of importance to more quantitative evaluation of the flow visualization is the determination of the airspeed or flow rate in the duct model. The airspeeds are too small for the normal wind tunnel measurement system which makes use of the pressure drop in the wind tunnel converging section resulting from the Bernoulli effect. An indirect method is possible, by measuring the volumetric flow rate supplied to the rake manifold from the smoke generation system. If the total volumetric flow rate of air 
containing smoke is $\mathrm{Q}$, then the average discharge velocity from $\mathrm{N}$ identical rake tubes will be $\mathrm{U}$ $=\mathrm{Q} /(\mathrm{NA})$, where $\mathrm{A}$ is the internal cross-sectional area of a single typical rake tube. Since the exit tube flow must be properly balanced with the external airspeed for so-called iso-kinetic injection, $U$ will be approximately the airspeed in the tunnel test section at the point of the smoke rake exit. Alternatively, a small rotating vane anemometer could possibly be placed in a neutral location, depending on the type of test section model configuration. For the current testing, the volumetric flow rate was less than about $10 \mathrm{SCFH}$ (280 liters/hour). The 1/4-inch 18-tube stainless steel rake tubes have an inside diameter of about 0.200 inches $(0.508 \mathrm{~cm})$, which yields a nominal airspeed of about $1 \mathrm{ft} / \mathrm{sec}(0.4 \mathrm{~m} / \mathrm{sec})$.

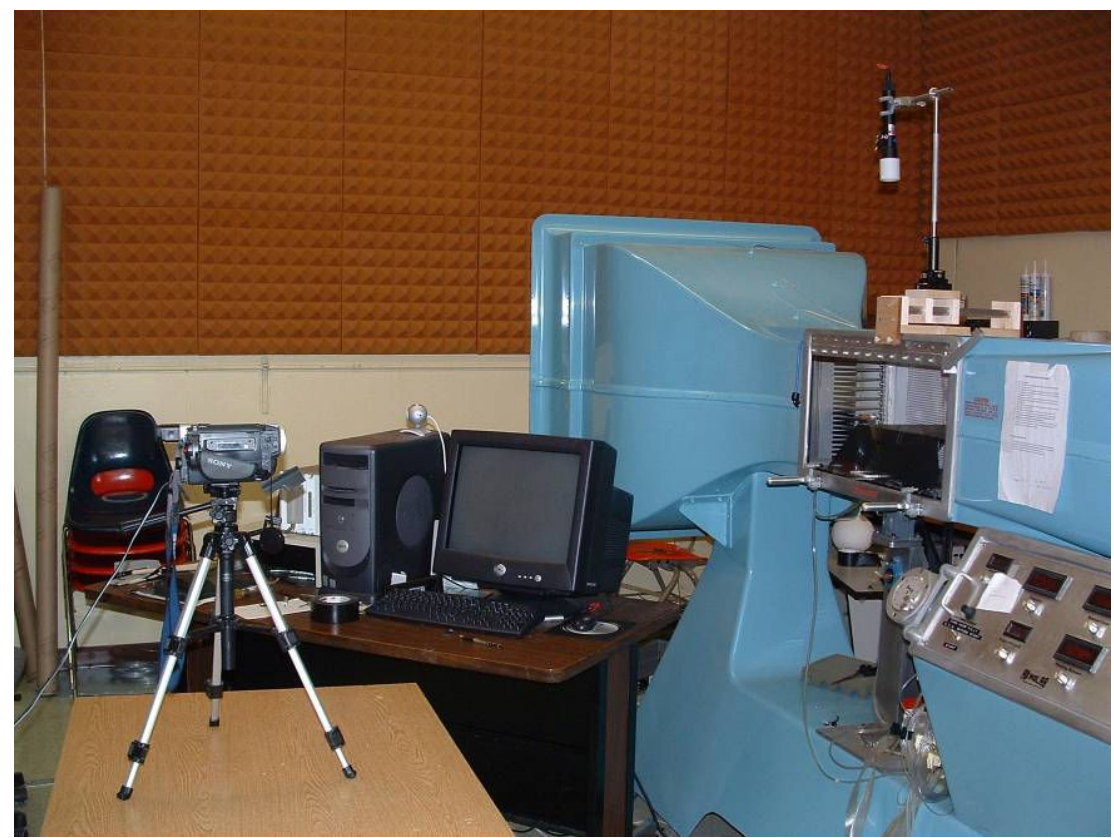

Figure 6: Overall Imaging System and Wind Tunnel Setup

\section{Streamline Illumination and Image Acquisition System}

For clear visualization of the smoke streams, an illumination system is also needed. Figures 5 and 6 also show the laser-based illumination setup used for the current testing. A 500mW solid state laser was mounted vertically on an adjustable support platform. The platform provided lateral displacement adjustment and tilt adjustment degrees of freedom for aligning the light sheet with the plane of the smoke streams. A simple cylindrical lens optic produced the desired sheet of light for illumination of a section of the test section. The top of the test section, as well as the side-walls, are of Plexiglas for optical access. Not all of the flow field could be viewed at the same time with the current optical setup due to the spreading of the laser light sheet. For safety reasons, precautions were taken to minimize stray laser reflections. The wing test model was painted flat black opaque for this reason. Figure 6 shows a photograph of the overall experimental setup, including the image acquisition setup. A generic camcorder with tripod mount was used to capture both video and single frame images of the streamline flow. The camcorder was positioned about $10 \mathrm{ft}(3 \mathrm{~m})$ from the image plane to minimize parallax effects and to also provide large depth of field to keep everything in focus. It should be noted that the camcorder is shown somewhat closer in the above Figure. The positioning traverse for the top- 
mounted illumination system has recently been improved over the prototype shown in Figure 6 .

\section{Flap Rotation Mechanism and Wing Model}

A wide variety of test section geometries can be implemented for visualization of flows using the above system. For the present honors project work, a simplified rotating flap system was designed and constructed so as to provide adjustable periodic rotation of the trailing edge flap. Figure 7(a) shows a schematic diagram of the mechanism used to rotate the wing trailing edge flap, and Figure 7(b) is a photograph of the actual prototype mechanism attached to the flap of the wing model. It was constructed from simple inexpensive materials available from most hardware stores, and was driven by a small DC electric motor with a gear reduction attachment. The rotation speed was such that rotation frequencies were on the order of $1 \mathrm{~Hz}$ or less. The linkages were configured so as to provide an angular flap deflection of about \pm 40 degrees.

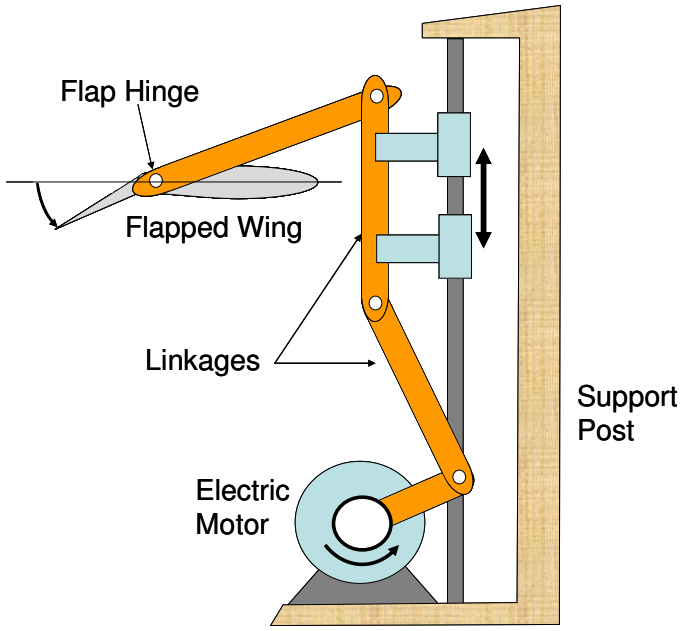

(a) Simplified Schematic

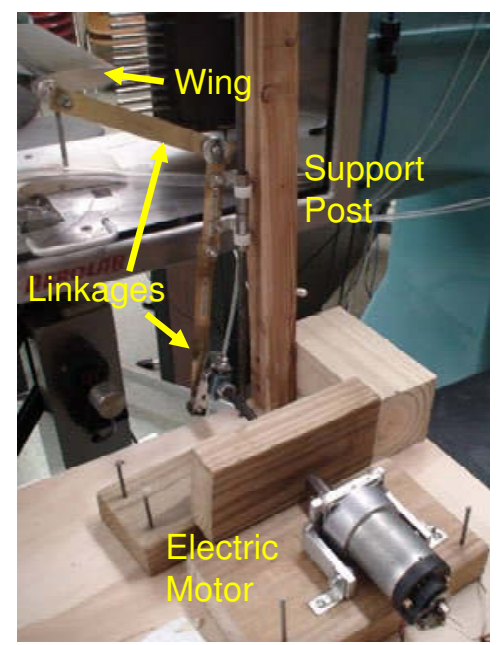

(b) Photograph of Rotating Mechanism

Figure 7: Wing Flap Rotation Mechanism

Figure 8 shows a CAD drawing of the wing model used in the current testing. It was fabricated using our rapid prototyping facility with a symmetrical NACA0030 airfoil shape, and had a chord length of 5.0 in $(75 \mathrm{~mm})$ and a $40 \%$ flap.

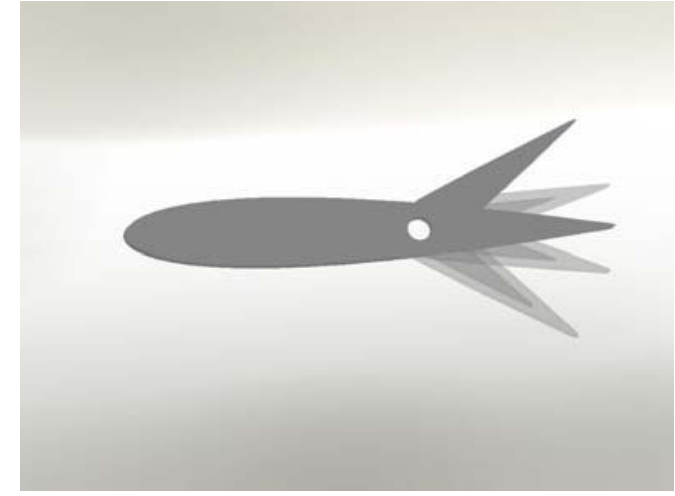

(a) Rotating Wing Flap Design

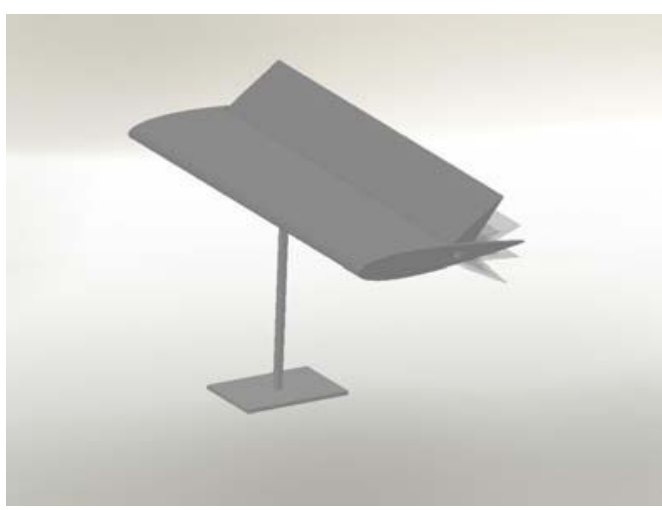

(b) 3D view of Wing and Support

Figure 8: CAD Drawing of Airfoil and Wing Model with Support 
The flap pivot was a solid shaft which extended out either side of the wind tunnel test section for support, and connected to the rotation mechanism on one side of the wind tunnel. Figure 8(a) shows an end view and illustrates the range of flap deflection possible. Figure 8(b) shows an isometric or 3D view of the entire flapped wing, along with the simple pedestal support for mounting in the wind tunnel. Figure 9(a) below shows a photograph of the flapped wing mounted in the wind tunnel, and Figure 9(b) shows the detail associated with the metal support plates and flap hinge.

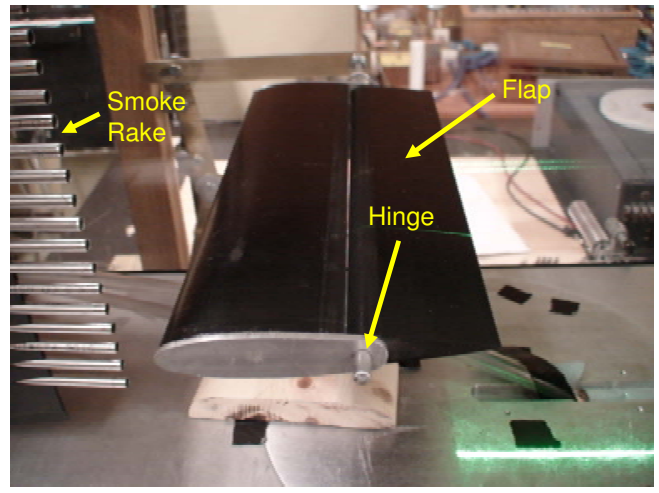

(a) Flapped Wing Model

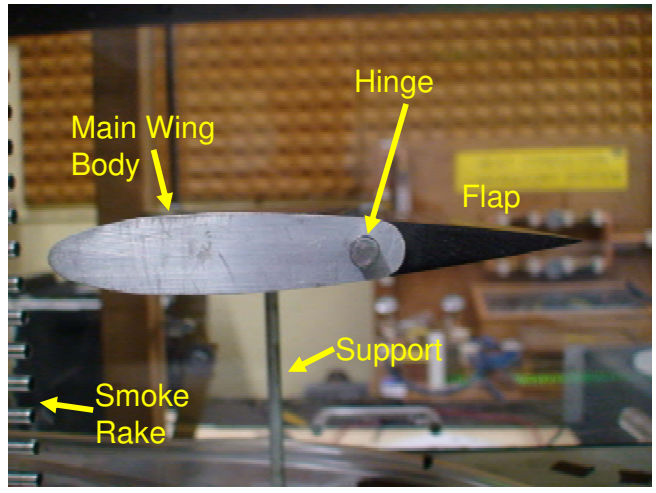

(b) End View of Flapped Wing

\section{Figure 9: Photographs of Flapped Wing in Wind Tunnel}

Air currents caused by the room air-distribution system or movement of individuals near the wind tunnel entrance or exit can significantly affect the steadiness of the streams. Slight ambient pressure changes in the room can affect the flows as well, such as might result from nearby doors opening or closing - even if not in the same room. With proper attention to these details, and with a little patience, it is relatively easy to achieve good results.

\section{Wing Morphing Test Model Flow Visualization Results}

Figure 10(a) shows a schematic diagram of the periodic vortex shedding from the periodic morphing wing shape, and Figure 10(b) shows an actual photograph of the flow visualization of the actual wing wake vortex shedding phenomena. The alternating vortices are very periodic and easily visible, although the field of view in the current setup is somewhat limited due to the limited light sheet illumination area. This is even more evident in a video of the wake region.

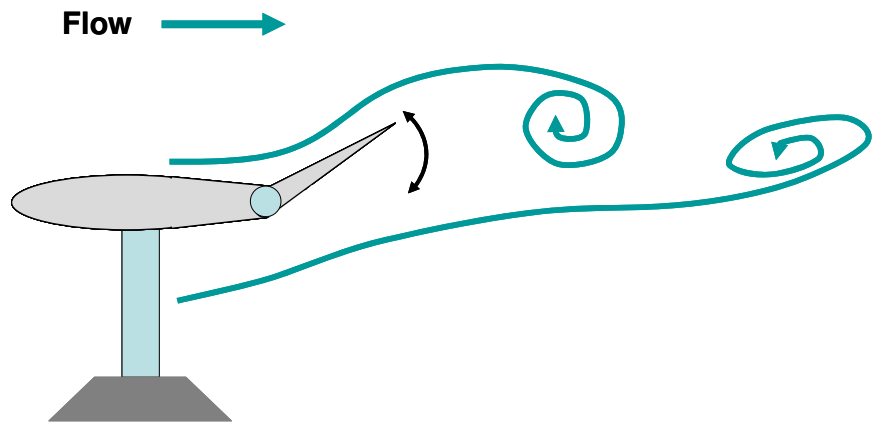

(a) Sketch of Periodic Vortex Shedding

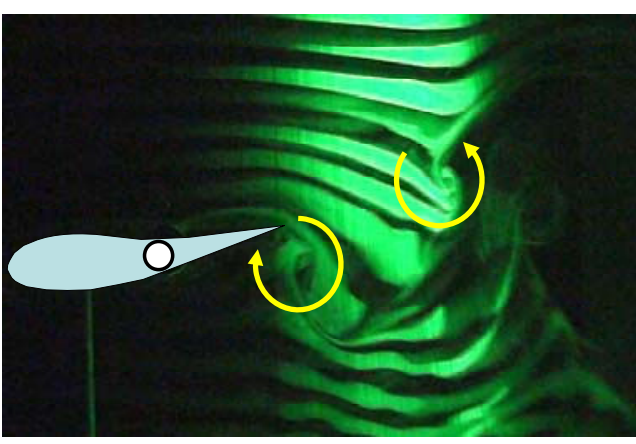

(b) Photograph of Vortex Shedding

Figure 10: Periodic Vortex Shedding from Morphing Wing 
The approximate location of the wing profile has been drawn in for Figure 10(b) to better define the flap position. In the future, the end of the wing will be better illuminated. Some meandering of the streams results in loss of illumination in adjacent streams in still-frame captures, but the flow characteristics are still clearly evident in live video. Spreading or dithering of the light sheet could be used to enhance the definition of the still-frame captures, or alternatively this could be done through the use of frame averaging. In addition, slightly better resolution might be possible thorough the use of somewhat smaller and more closely space streams. The modular design allows for different configurations depending on the physical size of the test model, and with rapid prototyping the time for model construction is fast-typically, just overnight. These test results, and the techniques presented here, should be very useful in providing physical insight in undergraduate fluid mechanics courses, as well as in more advanced courses dealing with boundary layer separation and boundary layer control. The possible course applications of the hardware discussed in this paper include laboratory enhancement for the undergraduate fluid mechanics laboratory, the introductory aerodynamics laboratory, and practical laboratory experience associated with more advanced graduate level boundary layer theory courses. Specific learning objectives associated with this type of laboratory experience include the following:

1. Introduce students to practical optical fluid flow visualization techniques.

2. Develop understanding of engineering fluid mechanics principles associated with wind tunnel smoke rake design.

3. Develop experimental understanding of laminar and turbulent jet flows associated with smoke injection into an airstream.

4. Introduce students to streamline flow over flapped and unflapped airfoil shapes.

5. Develop understanding of engineering fluid mechanics principles associated with boundary layer separation and vortex shedding from morphing wing shapes.

6. Develop understanding of the differences between steady and unsteady flow over an airfoil.

7. Foster an important hands-on wind tunnel laboratory experience.

\section{Summary and Conclusions}

This paper presents the authors recent experience with a one semester senior undergraduate mechanical engineering student honors research design project which made use of our Aerolab educational wind tunnel test facility, along with some newly developed flow visualization hardware. The paper has demonstrated some of basic characteristics of one type of wing morphing on a dynamic wing model inexpensively fabricated using a rapid prototyping facility. Flow visualization of vortex shedding phenomena was conducted for the dynamic wing morphing design. The pivoted wing structure test model was driven in periodic rotational motion by a mechanism designed by the associated honors student. The rotation frequency was on the order of $1 \mathrm{~Hz}$. The mechanical setup was used in conjunction with a recently developed special-purpose modular aerodynamic smoke rake system, and a relatively inexpensive lowpower laser-based lighting system, for visualization of the associated wing wake vortex shedding phenomena. The extension of this experimental setup to include quantitative measurements of pressure distribution is a logical next step. This can readily be accomplished with the rapid prototyping capability, and is a planned future activity. Future additions planned include the 
testing of more complex wing morphing, perhaps more focused on wing morphing as a means of boundary layer control, and refinement of the image illumination system to extend the field of view and clarity of image captures. A more flexible (i.e., two or more degree of freedom) sectioned wing model is envisioned as the next logical step, which would require more sophisticated internal joint control.

\section{Acknowledgements}

The MNE research engineer and instrument shop machinist Jason Selland is gratefully acknowledged for his work in conjunction with the rapid prototyping system, which was used to construct the smoke rake and flapped wing designs tested in this paper.

\section{Bibliography}

1. Beck, B. Terry and Anderson, Brian E., "The Wind Tunnel as a Practical Tool for the Demonstration of Engineering Fluid Mechanics and Principles of Aerodynamic Design," Paper Number AC 2007-2821, Proceedings of the ASEE 2007 Annual Conference \& Exposition, Honolulu, Hawaii, June 24-27, 2007.

2. Beck, B. Terry and Anderson, Brian E., Hosni, Mina; "A Simple Educational Wind Tunnel Setup for Visualization of Duct Flow Streamlines and Nozzle/Diffuser Boundary Layer Separation," Paper Number AC 2008-2117, Proceedings of the ASEE 2008 Annual Conference \& Exposition, Pittsburg, PA, June 23-25, 2008.

3. Photograph of Flying Bird: http://www.paulnoll.com/Oregon/Birds/wing-slow-flapping.html.

4. Funk, R., Parekh, D., Crittenden, T. and Glezer, A., Transient Separation Control using Pulse Combustion Actuation, 1st Flow Control conference, 24-26 June 2002, Paper\# AIAA 2002-3166.

5. NASA Langley Research Center, Fact Sheet\# FS-2000-06-52-LaRC.

6. McCormick, B. W., Aerodynamics, Aeronautics and Flight Mechanics, 2nd Edition, Wiley, pp. 86-109, 1995.

7. Grossman, K. R., Cybyk, B. Z., and VanWie, D. M., Sparkjet Actuators for Flow Control, 41st AIAA Aerospace Sciences Meeting \& Exhibit, 6-9 June 2003, Paper\# AIAA-2003-0057.

8. Beck, B. T., A. D. Cutler, J. P. Drummond, S. B. Jones, "A Resonant Pulse Detonation Actuator for High-Speed Boundary Layer Separation Control," 11th International Symposium On Flow Visualization, August 9-12, 2004, University of Notre Dame, Notre Dame, Indiana, USA, August 9-12, 2004.

9. Cutler, Andrew. D., Beck, B. Terry, Wilkes, Jennifer A., Drummond, J. Philip, Alderfer, David W. and Danehy, Paul M., "Development of a Pulsed Combustion Actuator For High-Speed Flow Control," Paper No. AIAA2005-1084, 43rd AIAA Aerospace Sciences Meeting and Exhibit, Reno, Nevada, 10 - 13 Jan 2005.

10. Wu, C.-J., Wang, L., and Wu, J.-Z., 2007, "Suppression of the von Karman Vortex Street behind a Circular Cylinder by a traveling Wave Generated by a Flexible Surface," Journal of Fluid Mechanics, Vol. 574, pp. 365391.

11. Beck, B. T., "A Modular Wing-Tail Airplane Configuration for the Educational Wind Tunnel Laboratory," Proceedings of the 2004 ASME International Mechanical Engineering Congress \& Exposition, Anaheim, California, November 13-19, 2004.

12. Beck, B. T. and Pratt, Nelson, "A Simple Device for Wind Tunnel Performance Testing of Small Scale Powered Propellers," presented at the 2005 ASME Fluids Engineering Summer Conference, Houston, Texas, June 19-23, 2005.

13. Beck, B. T., "Introduction to Aerodynamics: A Design/Build/Test Experience for Undergraduate Mechanical Engineering Students," presented at the ASEE Annual Conference \& Exposition, Chicago, Illinois, June 18-21, 2006. 